Article

\title{
Tie-Line Reserve Power Probability Margin for Day-Ahead Dispatching in Power Systems with High Proportion Renewable Power Sources
}

\author{
Yue Chen ${ }^{1, * \mathbb{D}}$, Zhizhong Guo ${ }^{1}$, Abebe Tilahun Tadie ${ }^{1}$, Hongbo $\mathrm{Li}^{2}$, Guizhong Wang ${ }^{2}$ and \\ Yingwei Hou ${ }^{2}$ \\ 1 School of Electrical Engineering and Automation, Harbin Institute of Technology, Harbin 150001, China; \\ zhizhonggzz@sina.com (Z.G.); abebetlhn2@hit.edu.cn (A.T.T.) \\ 2 Electric Power Research Institute of HITZ, Harbin Institute of Technology at Zhangjiakou, \\ Zhangjiakou 075000, China; lihongbo19790127@126.com (H.L.); wgz2003@sina.com (G.W.); \\ hyw422700015@163.com (Y.H.) \\ * Correspondence: ycchenyue@foxmail.com
}

Received: 16 October 2019; Accepted: 11 December 2019; Published: 12 December 2019

\begin{abstract}
In power systems with a high proportion of renewable power sources (PSHPRPSs), the power constraints of the tie-line may limit the ability of the reserve power to accommodate uncertain power generation, resulting in difficulties for the grid power balance. As uncertain power generation cannot be predicted accurately and in accordance with the law of probability and statistics, it is necessary to use a probability model to calculate the uncertain power of the tie-line. Here, day-ahead prediction error probability optimal power flow (DPEPOPF) is proposed to calculate the tie-line reserve power probability margin (TRPPM) in day-ahead dispatching. In day-ahead dispatching, TRPPM is reserved for real-time dispatching to accommodate uncertain power generation, so as to avoid tie-line power congestion. This study classifies the area of the grid based on the principle of area control error accommodation, and the DPEPOPF is divided into two categories: An inter-area day-ahead prediction error probability optimal power flow mathematical model, and an intra-area day-ahead prediction error probability optimal power flow mathematical model. The point estimate optimization algorithm was implemented in MATLAB 8.3.0.532 (R2014a) to calculate the TRPPM. The simulation results verify the accuracy of the model and effectively avoid power congestion of the tie-line.
\end{abstract}

Keywords: uncertain power generation; PSHPRPSs; day-ahead dispatching; dispatch control system; tie-line power constraint; DPEPOPF; TRPPM

\section{Introduction}

Renewable power sources such as wind and solar power have uncertainties characterized based on randomness and intermittence. Grids with a high proportion of uncertain power sources are power systems with a high proportion of renewable power sources (PSHPRPSs). PSHPRPSs have a large degree of uncertain power generation. In order to maintain grid power balance, the PSHPRPSs must have a dispatch control system (DCS) with power accommodation capability to accommodate uncertain power generation. Conventional power grids do not have any uncertain power sources, so the day-ahead prediction of load power is relatively accurate. Therefore, the power prediction error is within the regulation range of the automatic generation control (AGC); accordingly, there is no requirement for other power balance links. In contrast, because of the large proportion of uncertain power sources, the error in day-ahead power prediction for PSHPRPSs is large, and the AGC cannot balance the uncertain power unless a real-time scaled power balance link is added. For the grid DCS, 
some studies have suggested three time scale options (short-term dispatching, real-time dispatching, and AGC [1]), while others propose four time scale options (day-ahead dispatching, rolling power dispatching, real-time dispatching, and AGC [2]). Research on the DCS of PSHPRPSs has mainly focused on increasing the power balance link over a short time scale, and on modifying the generation plan step-by-step to accommodate uncertain power generation [3-8]. Considering that the presence of too many short time scale power balancing links will affect the efficiency of the grid DCS, this study has adopted a three time scale DCS with day-ahead dispatching, real-time dispatching, and AGC.

In the DCS of PSHPRPSs, the accommodation of uncertain power generation is based on power forecasting. The day-ahead dispatching plan is formulated according to the day-ahead power forecast. According to the uncertain power source power prediction error between the real-time and day-ahead predictions (i.e., the uncertain power generation), a real-time power generation plan is formulated to ensure that the power error after real-time accommodation is within the AGC accommodation range. To effectively accommodate uncertain power generation in real-time dispatching, the day-ahead dispatching needs to reserve sufficient reserve power for real-time dispatching, including controllable power sources [9-13], energy storage technology [14-16], and active load [17-20]. Uncertain power generation cannot be accurately predicted with current technology, whether or not the physical prediction method [21-23], statistical prediction method [24-27], or comprehensive prediction method [28-30] is used. Therefore, the reserved power capacity is uncertain.

To accommodate the power prediction error of renewable energy sources, some works have conducted uncertainty analysis research. Considering a small hydropower source with no reservoir, a day-ahead dispatching model was proposed in reference [31] for a distributed power grid system which contains several distributed generators, such as small hydropower and wind power sources, and energy storage systems. In the presence of renewable energy sources and storage devices, Papari et al. proposed a stochastic framework for the optimal operation and management of hybrid alternating current (AC)-direct current (DC) microgrids [32]. A multi-time scale robust economic dispatch strategy with a multi-source hybrid power system based on variable confidence levels was proposed to reduce the impact of the renewable energy power prediction uncertainty [33]. Additionally, Papari et al. also developed a new two-stage approach for accurate modeling and prediction of tidal current to utilize renewable energy sources with unreliable and intermittent characteristics [34]. Each of the above works has provided good progress towards accommodating the power prediction error of renewable energy sources. However, these literatures do not study this problem from the perspective of power grid DCS, which requires reserve power to accommodate the power prediction error of renewable energy sources. To avoid tie-line power congestion affecting the utilization of reserve power, it is necessary to reserve a tie-line power margin for reserve power in the day-ahead dispatching of PSHPRPSs. Therefore, in comparison with the existing literature, the main contributions of this work can be summarized as follows: (1) The tie-line reserve power probability margin (TRPPM) is set aside for reserve power in the day-ahead dispatching to avoid power congestion in real-time dispatching. (2) The power prediction error of renewable energy sources is accommodated from the perspective of grid DCS. (3) The day-ahead prediction error probability optimal power flow (DPEPOPF) can simplify the probabilistic optimal power flow model and improve its efficiency.

The day-ahead predicted errors of uncertain power generation conform to the laws of probability and statistics [35-37], and the tie-line reserve power margin for balancing day-ahead predicted errors conforms to probability and statistics laws. Therefore, the probability model is adopted in this study. Sensitivity is used to estimate the effect of parameter changes on the transfer capacity. This procedure is linearly approximate and quick [38]. Due to the large error of power forecasting, the day-ahead dispatching plan cannot be accurate, and the power prediction error is accommodated by real-time dispatching and AGC. On this basis, we developed a mathematical model of DPEPOPF, which solves the numerical characteristics of the TRPPM in day-ahead dispatching. Based on this, the tie-line reserve power probability margin can be determined, which lays a foundation for real-time dispatching to effectively accommodate uncertain power. 
The remainder of this paper is structured as follows. Section 2 presents the divided power grid according to the property of the power source. Section 3 presents the DPEPOPF mathematical model and its solution. Section 4 presents the simulation results for the DPEPOPF, as applied to the Institute of Electrical and Electronics Engineers (IEEE) 118 bus test system to calculate the TRPPM in the day-ahead dispatching. Finally, the conclusions of this work are presented in Section 5.

\section{Grid Area Division Based on Power Source Properties}

To reduce or avoid the impact of the power generation uncertainty on other grid areas, the uncertain power generation needs to be accommodated in a way that complies with the principle of area control error accommodation. The power generated from large uncertain power sources such as large wind and photovoltaic power stations needs to be transported via a tie-line to other grid areas where the load is concentrated. For large uncertain power sources that cannot be accommodated nearby, it is necessary to use the reserve power of other grid areas for accommodation. Based on the principle of area control error accommodation, the whole power system needs to be divided into several power grid areas.

\subsection{Types of Grid Area Division Based on Power Source Properties}

There are many methods for dividing the power grid area. According to the property of the power source in the grid areas, this study divides them into three types: A type I grid not only contains an uncertain power sources, but also has accommodation capacity. A type II grid is a power system with only uncertain power sources. A type III grid is a power system without an uncertain power sources, but which has the ability to accommodate uncertain power generation. In addition, other factors such as the uncertain power source correlation and geographical distance should also be considered when dividing a power grid area. After division, uncertain power sources in the same power grid area have greater correlations, while uncertain power sources in different power grid areas have lesser correlations.

According to the property of power supply, a power grid can be divided into power grid areas of types I, II, and III. For example, the power system shown in Figure 1a consists of five grids: One type I, one type II, and three type III grids. Each power grid area is equivalent to a power source and a load. The equivalent diagram of the power grid area is shown in Figure $1 b$.

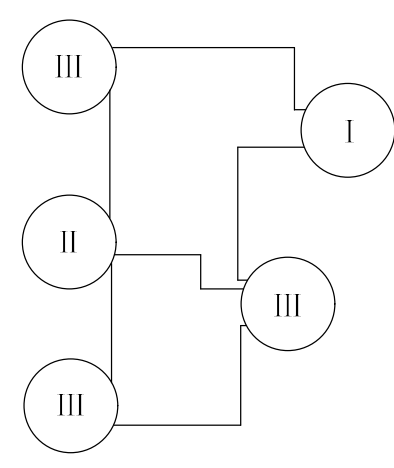

(a)

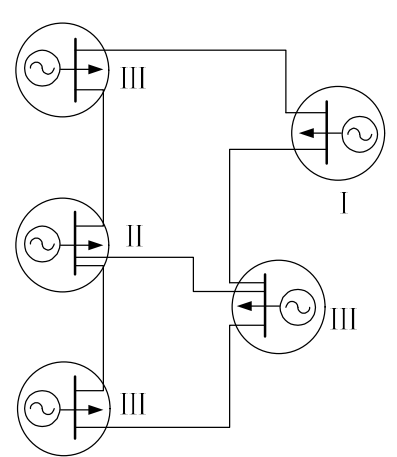

(b)

Figure 1. Schematic diagram of the power grid area division process. (a) Power grid area schematic diagram; (b) equivalent grid area schematic diagram.

\subsection{Equivalent Parameters of Power Grid Area}

Equivalent parameters of a power grid area can be divided into internal equivalence parameters and tie-line equivalence parameters. The equivalent power grid with $M$ buses is shown in Figure 2 [39]. According to the principle of radial equivalent independent (REI) equivalence, the grid area containing $M$ buses is equivalent to a single equivalent bus $M_{l}$. 


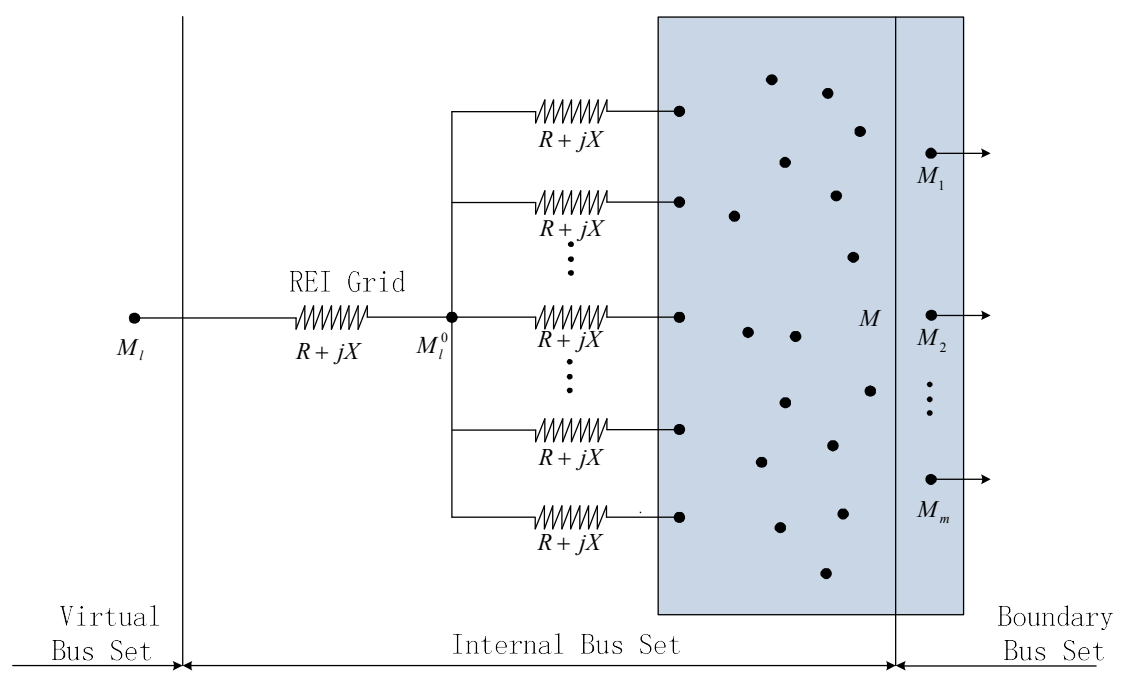

Figure 2. Diagram of an equivalent power grid.

For the grid area with $G$ power sources, the total active power of the grid is equivalent to the active power of equivalent bus $M_{l}$. The active power $P_{g}$ of the equivalent bus $M_{l}$ is defined by Equation (1):

$$
P_{g}=\sum_{i=1}^{G} P_{g, i}
$$

where $P_{g, i}$ is the active power of each power source.

For the grid area with $D$ loads, the total load of the grid is equivalent to the load power of equivalent bus $M_{l}$. The load power $P_{l}$ of the equivalent bus $M_{l}$ is defined by Equation (2):

$$
P_{l}=\sum_{i=1}^{D} P_{l, i}
$$

where $P_{l, i}$ is the power of each load.

Generally, there are several tie-lines between the two power grid areas, as shown in Figure 3.

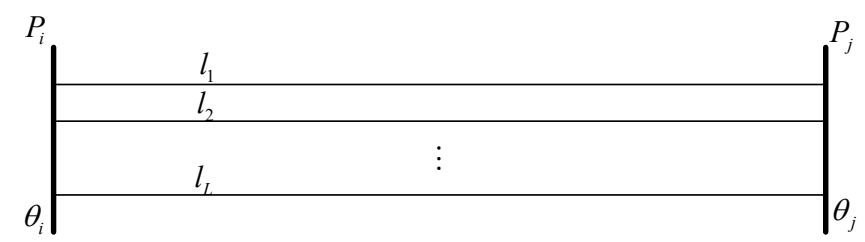

Figure 3. Inter-area tie-lines between two grids in the power grid area.

After the power grid area is equivalent to an equivalent bus. As shown in Figure 3, there are $L$ tie-lines between the two equivalent buses; the reactance of the $t$ th tie-line is $X_{i j, t}$. The equivalent active powers of the two equivalent buses are $P_{i}$ and $P_{j}$; the equivalent phase angles of two equivalent buses are $\theta_{i}$ and $\theta_{j}$.

According to the DC power flow model, the active power of $t$ th tie-line is defined by Equation (3):

$$
P_{i j, t}=\frac{\theta_{i}-\theta_{j}}{X_{i j, t}}, t=1,2, \cdots L
$$


The tie-line power between two equivalent buses is the sum of each tie-line power. The total active power of the tie-line between the two equivalent buses is defined by Equation (4):

$$
P_{i j}=P_{i j, 1}+P_{i j, 2}+\cdots+P_{i j, t}
$$

where $P_{i j}$ is the total power of tie-line; $P_{i j, t}$ is the power of $t$ th tie-line.

According to Equations (3) and (4), the total reactance between the two equivalent buses can be calculated. Therefore, the equivalent parameter of the tie-line between two equivalent buses is defined by Equation (5):

$$
X_{i j}=\frac{1}{\sum_{t=1}^{L} \frac{1}{X_{i j, t}}}
$$

\section{Prediction Error Probability Optimal Power Flow for Day-Ahead Dispatching}

The day-ahead dispatching power consists of the day-ahead power prediction value and power prediction error. According to the power predicted value of the day-ahead dispatching, the day-ahead power generation plan is formulated. According to historical statistical data for power prediction error, the reserve power is set. To avoid tie-line power congestion, the day-ahead dispatching needs to reserve the TRPPM for the reserve power. Previous research has shown that uncertain power generation obeys probability distributions [40-42], and many studies assume that the day-ahead power prediction error of uncertain power source obeys the normal distribution [43-45]. Given this context, this section proposes the DPEPOPF and its optimization algorithm to solve the TRPPM.

\subsection{DPEPOPF Based on Grid Area Division}

Uncertain power generation affects the amplitude and frequency of voltage. To avoid a larger area being affected by the uncertain power generation, the uncertain power generation should be accommodated for in the area control. Therefore, this study divides the power grid and accommodates the uncertain power generation in its own power grid area based on the principle of area control error accommodation to avoid affecting other power grid areas. Based on the power grid division, this study divides the tie-lines into inter-area tie-lines and intra-area tie-lines. In China, renewable energy sources and main loads are generally distributed in different power grid areas. Therefore, the tie-lines between renewable energy sources and main loads are important objects of our research. For this reason, we need to study the reserve power margin of the inter-area tie-line. On this basis, the DPEPOPF is divided into two models: An inter-area DPEPOPF mathematical model, and an intra-area DPEPOPF mathematical model. The TRPPM can be divided into the inter-area TRPPM and intra-area TRPPM. In this study, the inter-area DPEPOPF and intra-area DPEPOPF methods are used to calculate the inter-area TRPPM and the intra-area TRPPM, respectively.

Based on the principle of area control error accommodation, the power grid area first carries out self-accommodation, and then carries out a grid equivalent calculation. For the equivalent grid area, the inter-area TRPPM is calculated by the inter-area DPEPOPF. The calculation value of inter-area TRPPM is taken as the original data of the buses at both ends of the tie-line, and is brought into the intra-area day-ahead DPEPOPF to calculate the intra-area TRPPM. The calculation flow chart is shown in Figure 4. 


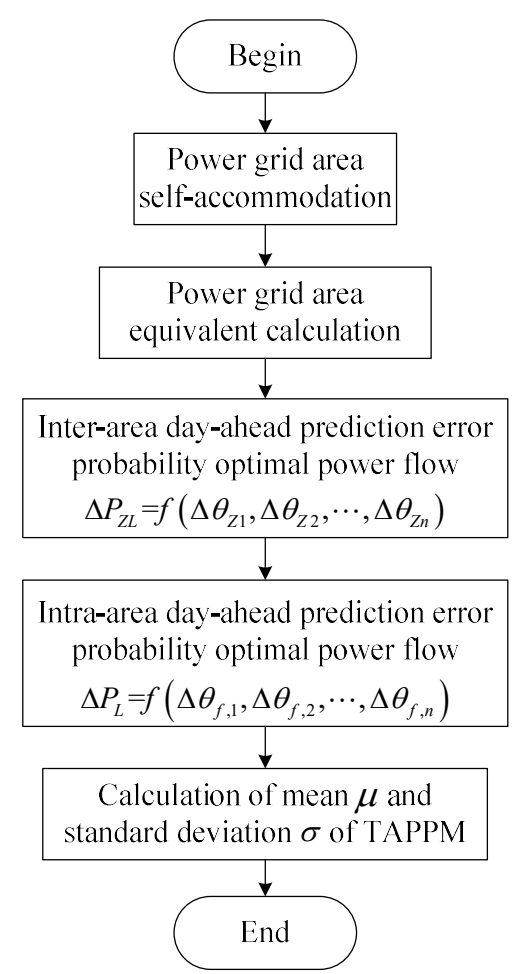

Figure 4. Flow chart of day-ahead prediction error probability optimal power flow (DPEPOPF) calculation.

\subsection{Inter-Area DPEPOPF}

\subsubsection{Prediction Error Probability Optimal Power Flow Mathematical Model}

Active power distribution is mainly considered in day-ahead dispatching. Active power is significantly affected by the phase angle of the bus voltage. To calculate the line power, the phase angle of bus voltage should be calculated first.

For a PSHPRPSs that can be divided into $n$ power grid areas, each power grid area is equivalent to a single bus. The power variation of equivalent buses caused by uncertain power generation is defined by Equation (6):

$$
\Delta P_{Z I}=B_{Z} \cdot \Delta \theta_{Z I}
$$

where $\Delta P_{Z I}$ is an $n-1$ dimensional variable representing the power variation of equivalent bus. The $\Delta P_{Z I}$ vector element is defined by Equation (7):

$$
\Delta P_{Z I}=P_{Z S}-P_{Z I}^{0}
$$

where $P_{Z S}$ is the active power of the equivalent bus; $P_{Z I}^{0}$ is the calculated value of equivalent bus active power. $P_{Z I}^{0}$ is calculated using AC power flow equations, which can reduce the error in the calculation of $\Delta P_{Z I}$.

The variable $\Delta \theta_{Z I}$ is $n-1$ dimensional and represents the phase angle variable of the equivalent bus. The voltage phase angle error calculated by the modified active power error is more accurate. 
The coefficient matrix $B_{Z}$ is an $n-1 \times n-1$ dimensional matrix whose elements are as shown in Equation (8), where $x_{i j}$ is the inter-area tie-line equivalent parameter calculated according to Equation (5).

$$
\left\{\begin{array}{c}
B_{i j}=-\frac{1}{x_{i j}} \\
B_{i i}=\sum_{\substack{j \in i \\
j \neq i}} \frac{1}{x_{i j}}
\end{array}\right.
$$

Since the $B_{Z}$ matrix is an invertible matrix, Equation (6) is transformed into Equation (9):

$$
\Delta \theta_{Z I}=B_{Z}^{-1} \cdot \Delta P_{Z I}=\mathrm{T}_{Z} \cdot \Delta P_{Z I}
$$

For any branch $l$, the from-bus is the $i$ bus, and the to-bus is the $j$ bus. The active power variation of the line between bus $i$ and bus $j$ is defined by Equation (10):

$$
\Delta P_{Z, i j}=V_{i}^{2} \ddot{y}_{i 0}^{*}+V_{i}^{2} G_{i j}-V_{i} V_{j}\left(G_{i j} \cos \Delta \theta_{i j}+B_{i j} \sin \Delta \theta_{i j}\right)
$$

where $y_{i 0}$ represents the admittance between bus $i$ and the zero-potential bus; $G_{i j}$ represents the conductance between bus $i$ and bus $j$; and $B_{i j}$ represents the reactance between bus $i$ and bus $j$.

According to Equation (10), Equation (11) is used to calculate the power margin of each inter-area tie-line.

$$
\Delta P_{Z L}=f\left(\Delta \theta_{Z 1}, \Delta \theta_{Z 2}, \cdots, \Delta \theta_{Z n}\right)
$$

When the power of the tie-line between power grid areas exceeds the upper limit, it is necessary to adjust this power. For the PSHPRPSs, which can be divided into $n$ power grid areas, the tie-line power adjustment mathematical model is given by Equation (12). This equation satisfies the line power constraint.

$$
P_{Z L}{ }^{\prime}=f\left(\theta_{Z 1}{ }^{\prime}, \theta_{Z 2}{ }^{\prime}, \cdots, \theta_{Z n}{ }^{\prime}\right)
$$

In order to avoid power congestion of tie-line between power grid areas, the maximum value of tie-line reserve power probability margin is selected. The inter-area day-ahead prediction error probability optimal power flow mathematical model is defined by Equation (13):

$$
\max F\left(P_{Z R, i}\right)=\max \sum \Delta P_{Z l, i}
$$

The intra-area day-ahead prediction error probability optimal power flow mathematical model of PSHPRPSs is constrained by power balance constraints, tie-line power constraints, and reserve capacity constraints, as described and quantified below.

(1) Power balance constraint

$$
\sum \Delta P_{f}+\sum P_{r}=0
$$

Equation (14) is a power balance constraint formula, where $\sum \Delta P_{f}$ denotes the day-ahead power prediction error and $\sum P_{r}$ denotes the reserve power.

(2) Tie-line power constraint

The active power variation $\Delta P_{Z l, i}$ of the power grid area tie-line should not be greater than the line residual power margin $\Delta P_{Z l, i m a x}$. The constraint is expressed as shown in Equation (15):

$$
\Delta P_{Z l, i} \leq \Delta P_{Z l, i \max }, i=1,2, \cdots, L
$$


(3) Reserve capacity constraint

The variable $P_{Z R, i}$ denotes the reserve power of the $i$ th power grid area, which satisfies the capacity constraint shown in Equation (16):

$$
P_{Z R, i \min } \leq P_{Z R, i} \leq P_{Z R, i \max }, i=1,2, \cdots r
$$

Type I and type III power grids have the capacity of accommodation. According to the principle of area control error accommodation, uncertain power generation should avoid flow to the external power grid. Therefore, a type I power grid should be used first to accommodate uncertain power generation. The accommodation capacity of a type I grid is $\sum P_{R, I}$ and that of a type III grid is $\sum P_{R, I I I}$. The variable $g\left(P_{Z R, i}\right)$ denotes the reserve power function and is defined by Equation (17):

$$
g\left(P_{Z R, i}\right)=\left\{\begin{array}{cc}
\sum P_{Z R, I} & , \text { if } \sum P_{Z R, i} \leq \sum P_{Z R, I} \\
\sum P_{Z R, I}+\sum P_{Z R, I I I} & \text { if } \sum P_{Z R, i} \leq \sum P_{Z R, I}+\sum P_{Z R, I I I}
\end{array}\right.
$$

\subsubsection{Inter-Area Point Estimation Optimization Algorithm for DPEPOPF}

The tie-line power variation $\Delta P_{Z L}$ and each value of power grid area uncertainty power generation power $\Delta P_{Z f}$ satisfy the functional relationship shown in Equation (18):

$$
\Delta P_{Z L}=F\left(\Delta P_{Z f 1}, \Delta P_{Z f 2}, \cdots, \Delta P_{Z f i}, \cdots, \Delta P_{Z f n}\right)
$$

where $\Delta P_{Z f i}$ represents the uncertain power generation of the $i$ th regional power grid; the mean is $\mu_{Z i}$ and the standard deviation is $\sigma_{Z i}$. The value of $\Delta P_{Z f i, j}$ is calculated using Equation (19) [46]:

$$
\Delta P_{Z f i, j}=\mu_{Z i}+\eta_{Z i, j} \cdot \sigma_{Z i}
$$

where $\eta_{Z i, j}$ denotes the standard location coefficient, which is calculated using Equation (20):

$$
\eta_{Z i, j}=\left\{\begin{array}{cc}
\frac{\lambda_{i, 3}}{2}+(-1)^{3-j} \cdot \sqrt{\lambda_{i, 4}-\frac{3}{4} \cdot \lambda_{i, 3}^{2}} & , j=1,2 \\
0 & , j=3
\end{array}\right.
$$

where $\lambda$ is calculated using the ratio of central moments $M\left(\Delta P_{Z f i}\right)$ to standard deviation $\sigma_{Z i}$. The $M\left(\Delta P_{Z f i}\right)$ value is calculated using Equation (21) and $\lambda$ using Equation (22):

$$
\begin{aligned}
M_{t}\left(\Delta P_{Z f i}\right) & =\int_{-\infty}^{+\infty}\left(\Delta P_{Z f i}-\mu_{Z i}\right)^{t} \cdot f\left(\Delta P_{Z f i}\right) d \Delta P_{Z f i} \\
\lambda_{i, t} & =\frac{M_{t}\left(\Delta P_{Z f i}\right)}{\left(\sigma_{Z i}\right)^{t}}, t=1,2,3, \cdots, n
\end{aligned}
$$

In Equation (21), $f\left(\Delta P_{Z f i}\right)$ represents the probability density function (PDF) of the power grid area uncertainty power generation.

According to Equation (18), if the power prediction error of uncertainty power source $\Delta P_{Z f}$ obeys the probability distribution, the inter-area tie-line power variation $\Delta P_{Z L}$ will also obey the probability distribution. Here, the point estimation optimization algorithm for inter-area DPEPOPF is used to obtain the numerical characteristics of inter-area tie-line power variation $\Delta P_{Z L}$. The calculation steps of the point estimation optimization algorithm are as follows: 
(1) According to Equation (23), the inter-area tie-line power $P_{Z L}$ is calculated using the power grid area uncertainty power source predicted value:

$$
P_{\mathrm{ZL}}=f\left(\theta_{\mathrm{Z} 1}, \theta_{\mathrm{Z} 2}, \cdots, \theta_{\mathrm{Zn}}\right)
$$

(2) Whether any tie-line exceeds the upper limit power is detected according to Equation (24); if the upper limit is exceeded, the third step will be executed; otherwise, the fourth step will be executed:

$$
P_{Z l} \leq P_{Z l \max }, i=1,2, \cdots, L
$$

(3) According to Equation (12), the mathematical model of the inter-area tie-line power adjustment power flow is used to adjust the tie-line power.

(4) The inter-area tie-line residual power margin is calculated according to Equation (25):

$$
\Delta P_{Z l \max }=P_{Z l \max }-P_{Z l}
$$

(5) According to the point estimation optimization algorithm for the DPEPOPF, the variables $\Delta P_{Z f i}$ are selected in turn.

(6) The power grid area uncertain power generation probability variable is updated according to Equation (19).

(7) After the power grid area is self-accommodated, the residual power margin $\Delta P_{Z f i}{ }^{\prime}$ of power grid area is calculated.

(8) According to Equation (11), the inter-area DPEPOPF is calculated.

(9) The mean $\mu_{Z l}$ and standard deviation $\sigma_{Z l}$ of the inter-area TRPPM are calculated.

A flow chart of the inter-area point estimation optimization algorithm for DPEPOPF is shown in Figure 5. 


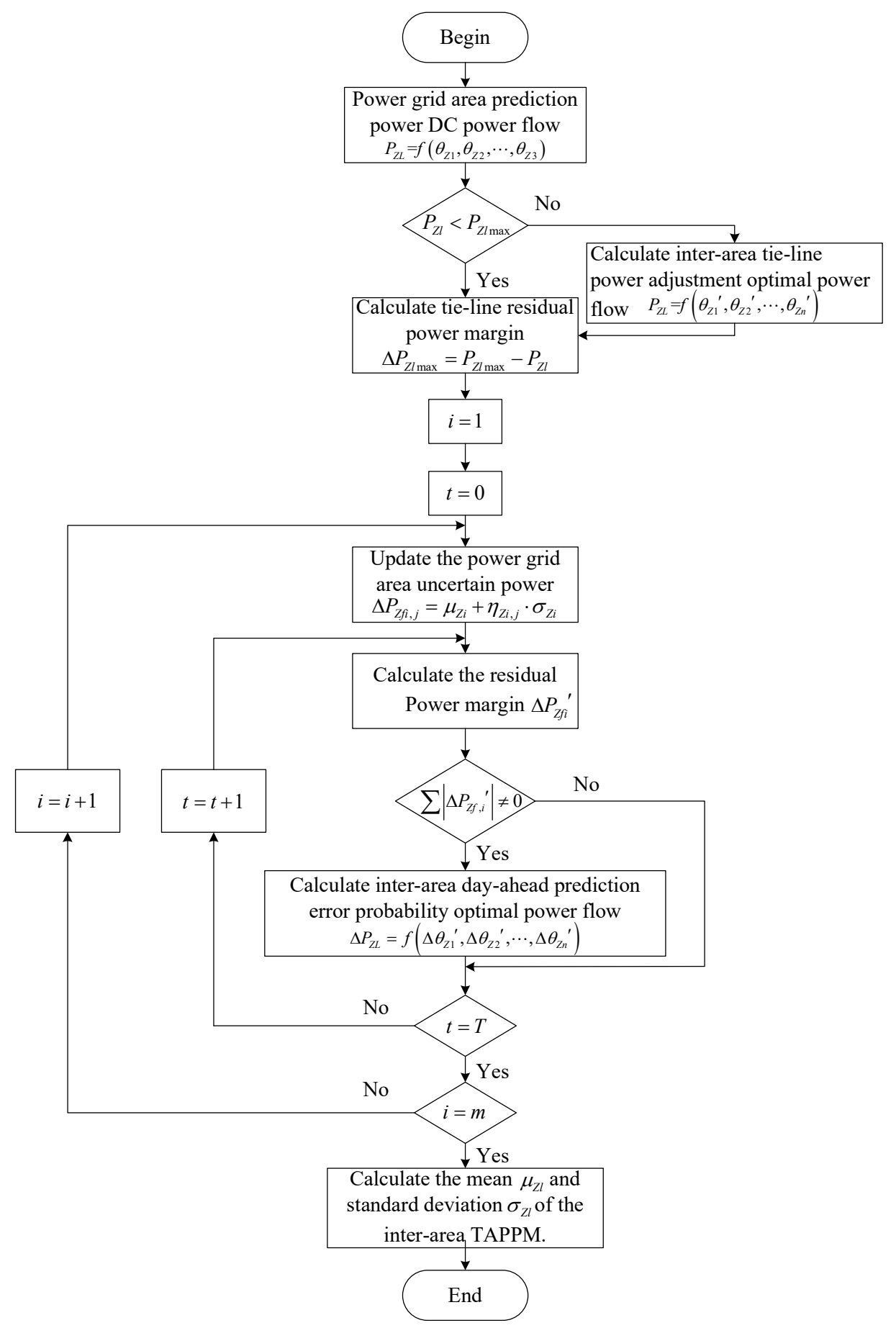

Figure 5. Flow chart of the inter-area point estimation optimization algorithm for DPEPOPF.

\subsection{Intra-Area DPEPOPF}

\subsubsection{Prediction Error Probability Optimal Power Flow Mathematical Model}

For a PSHPRPS with $n$ buses, the bus power variation caused by uncertain power generation is defined by Equation (26):

$$
\Delta P_{I}=B \cdot \Delta \theta_{I}
$$


where $\Delta P_{I}$ is an $n-1$ dimensional variable representing the bus power variation. The $\Delta P_{I}$ vector element is defined by Equation (27):

$$
\Delta P_{I}=P_{S}-P_{I}^{0}
$$

where $P_{S}$ is the bus active power, and $P_{I}^{0}$ is the initially calculated bus active power. $P_{I}^{0}$ is calculated using AC power flow equations, which can reduce the error in the $\Delta P_{I}$. The $\Delta \theta_{I}$ is an $n-1$ dimensional variable representing the bus phase angle. The voltage phase angle error calculated using the modified active power error is more accurate. The coefficient matrix $B$ is an $n-1 \times n-1$ dimensional matrix whose elements are as shown in Equation (8).

According to Equation (10), Equation (28) is used to calculate the power margin of each intra-area tie-line.

$$
\Delta P_{L}=f\left(\Delta \theta_{1}, \Delta \theta_{2}, \cdots, \Delta \theta_{n}\right)
$$

When the power of the tie-line exceeds the upper limit, it is necessary to adjust the tie-line power. For a PSHPRPS with $N$ buses, the mathematical model of the tie-line power adjustment is given by Equation (29). This equation satisfies the line power constraint.

$$
P_{L}{ }^{\prime}=f\left(\theta_{1}{ }^{\prime}, \theta_{2}{ }^{\prime}, \cdots, \theta_{n}{ }^{\prime}\right)
$$

To avoid tie-line power congestion, the maximum value of the tie-line reserve power probability margin is selected. The intra-area DPEPOPF mathematical model is defined by Equation (30):

$$
\max \mathrm{F}\left(P_{R, i}\right)=\max \sum \Delta P_{l, i}
$$

The intra-area DPEPOPF mathematical model of PSHPRPSs is constrained by power balance constraints, tie-line power constraints, and reserve capacity constraints, as described and quantified below.

(1) Power balance constraint

Equation (31) is an expression of the power balance constraint:

$$
\sum \Delta P_{I}+\sum P_{R, I}=0
$$

where $\sum \Delta P_{I}$ represents the intra-area uncertain generating power; and $\sum P_{R, I}$ represents total reserve power.

(2) Tie-line power constraint

The active power variation $\Delta P_{l, i}$ of the power grid area tie-line should not be greater than the line residual power margin $\Delta P_{l, \text { imax }}$. The constraint is expressed by Equation (32):

$$
\Delta P_{l, i} \leq \Delta P_{l, \text { imax }}, i=1,2, \cdots, L
$$

(3) Reserve capacity constraint

The number of reserve power sources in the power grid is $r$, and $P_{R, i}$ represents the reserve power generation power, which satisfies the reserve capacity constraint shown in Equation (33):

$$
P_{R, i \min } \leq P_{R, i} \leq P_{R, i \max }, i=1,2, \cdots r
$$

\subsubsection{Intra-Area DPEPOPF}

Uncertain power generation $\Delta P_{f}$ obeys the probability distribution. The tie-line power variation $\Delta P_{L}$ and uncertainty power generation power $\Delta P_{f}$ satisfy the functional relationship shown in Equation (34):

$$
\Delta P_{L}=F\left(\Delta P_{f, 1}, \Delta P_{f, 2}, \cdots, \Delta P_{f, i}, \cdots, \Delta P_{f, n}\right)
$$


where $\Delta P_{f, i}$ represents the $i$ th uncertain power generation variation; the mean is $\mu_{i}$, and the standard deviation is $\sigma_{i}$. According to the principle of power grid area division, the uncertain power sources in the same power grid area are highly correlated, and $\Delta P_{f, i}$ is calculated using Equation (35):

$$
\Delta P_{f, i}=\mu_{i}+\lambda \cdot \sigma_{i}, \lambda=0,1
$$

According to Equation (34), the generation power uncertainty $\Delta P_{f}$ will lead to tie-line power variation $\Delta P_{L}$ obeying the probability distribution. Here, the intra-area DPEPOPF optimization algorithm is used to obtain the numerical characteristics of intra-area tie-line power variation $\Delta P_{L}$. The calculation steps are as follows:

(1) According to Equation (36), the tie-line power $P_{L}$ is calculated using the uncertainty power source predicted value:

$$
P_{L}=f\left(\theta_{1}, \theta_{2}, \cdots, \theta_{n}\right)
$$

(2) Detecting whether each tie-line power exceeds the upper limit according to Equation (37). If the upper limit is exceeded, the third step will be executed, otherwise the fourth step will be executed.

$$
P_{l} \leq P_{l \max }
$$

(3) According to Equation (29), a mathematical model of the intra-area tie-line power adjustment power flow is used to adjust the tie-line power.

(4) Each value of the tie-line residual power margin is calculated according to Equation (38):

$$
\Delta P_{l \max }=P_{l \max }-P_{l}
$$

(5) Update the uncertain power generation probability variable according to Equation (35).

(6) According to Equation (28), the intra-area DPEPOPF is calculated.

(7) Calculate the mean $\mu_{l}$ and standard deviation $\sigma_{l}$ of the intra-area TRPPM.

A flow chart of the intra-area the DPEPOPF optimization algorithm is shown in Figure 6. 


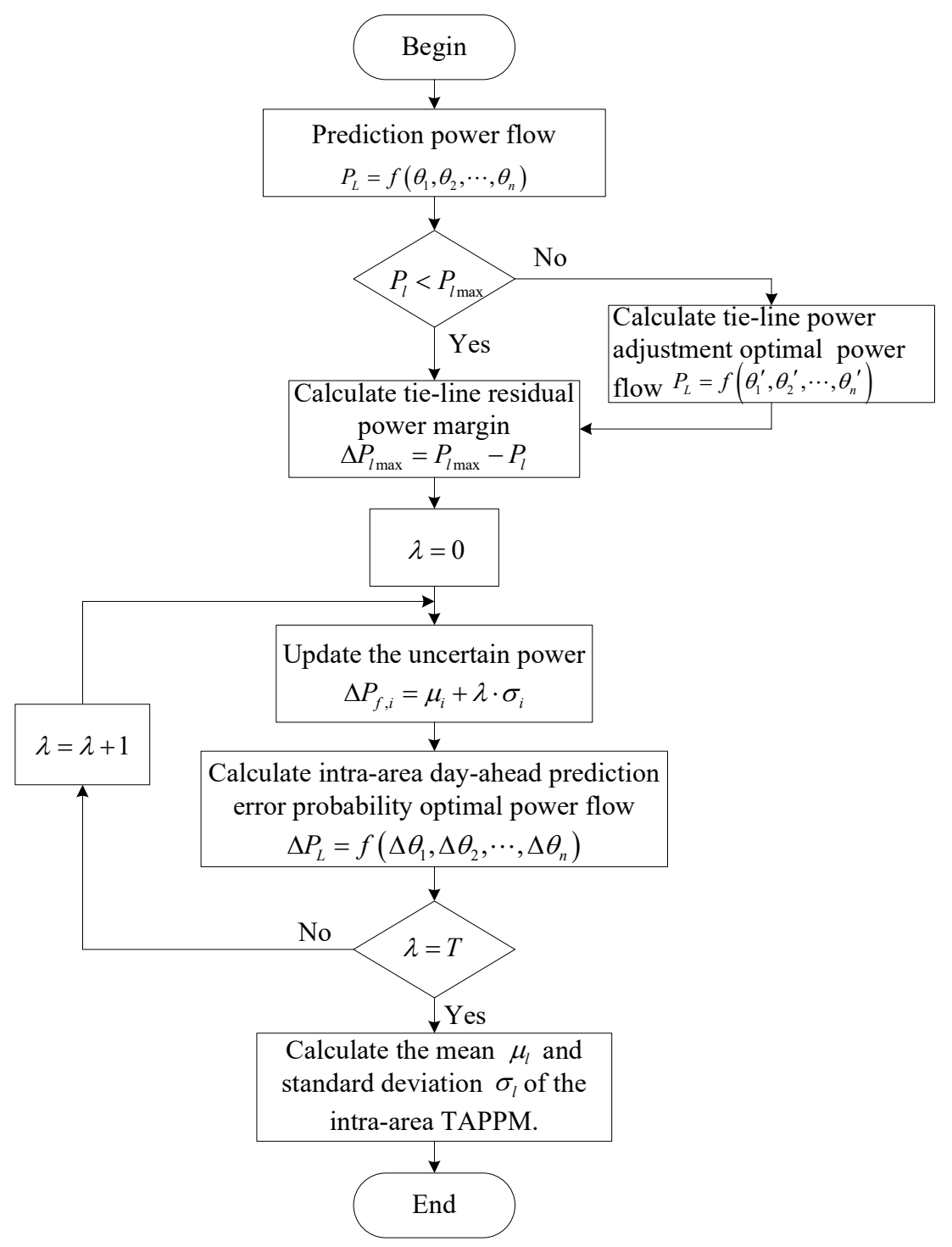

Figure 6. Flow chart of the intra-area DPEPOPF optimization algorithm.

\section{Simulation Results}

\subsection{Modified IEEE 118 Bus Test System Raw Data}

To verify the feasibility of the model, the DPEPOPF is applied to the modified IEEE 118 bus test system to calculate the TRPPM for the day-ahead dispatching. The modified IEEE 118 bus test system diagram is shown in Figure 7. Simulations were performed in MATLAB 8.3.0.532 (R2014a, The MathWorks, Inc, Natick, MA, USA) on a personal computer with an i3-4170 CPU, 3.70 GHz processor, and 4.0 GB RAM. 


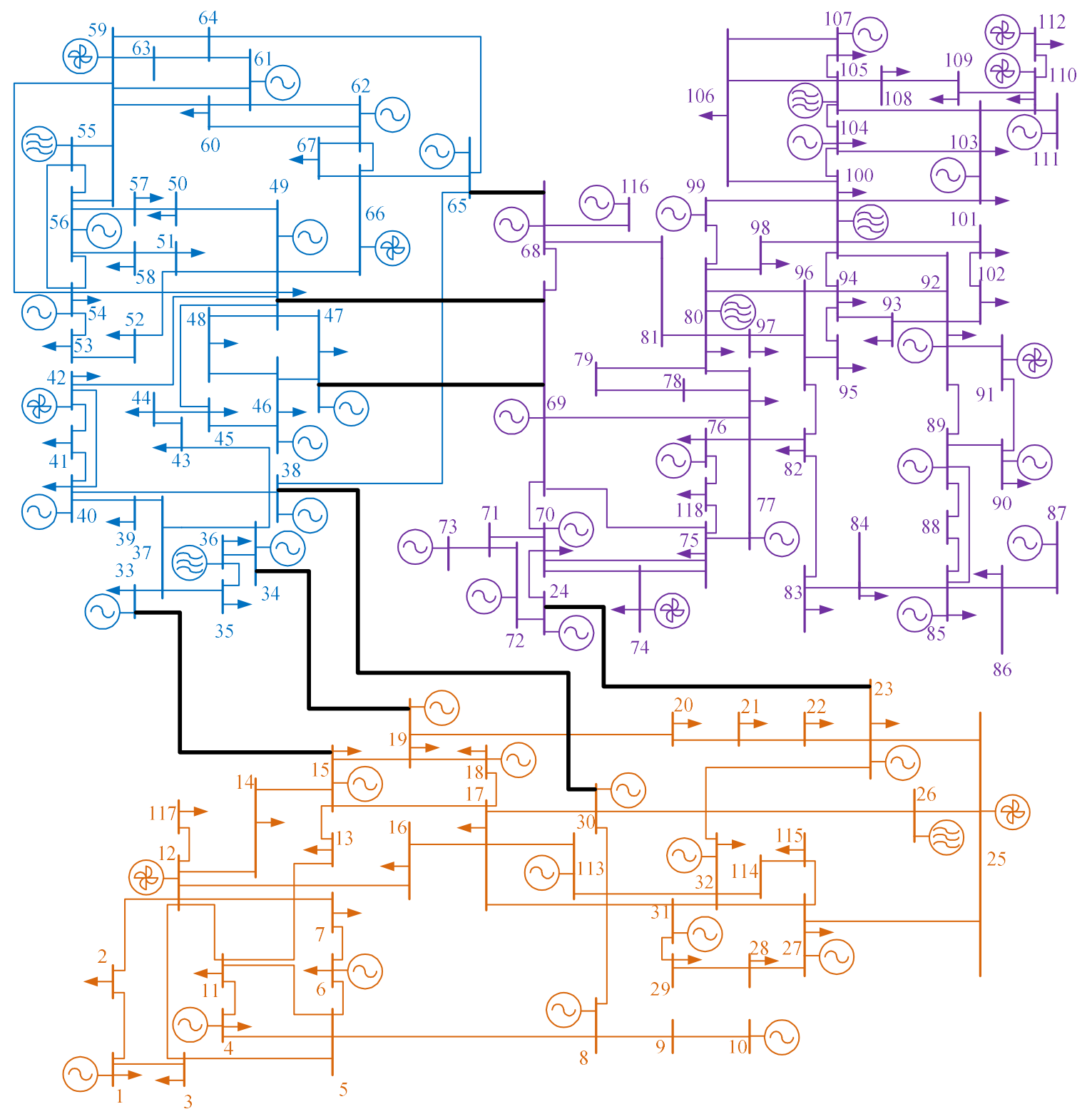

Figure 7. Modified Institute of Electrical and Electronics Engineers (IEEE) 118 bus test system diagram. The orange power area grid is area 1; the blue power area grid is area 2; and the purple power area grid is area 3. The thick black line is the inter-area tie-line.

According to the principles of the power grid area division explained in Section 2, this study divides the modified IEEE 118 bus test system into three power grid areas [47]. The mean and standard deviation of the uncertain power source prediction error are shown in Table A1.

Here, the DPEPOPF is used to calculate the TRPPM for day-ahead dispatching. Uncertainty power source power prediction error obeying the probability distribution will lead to tie-line power variation obeying the probability distribution. In the day-ahead dispatch, the tie-line needs to reserve the TRPPM for reserve power. According to the PDF, the probability values corresponding to the different TRPPM can be obtained.

\subsection{Simulation Results of Inter-Area TRPPM}

According to Equation (11), the inter-area DPEPOPF mathematical model is used to solve the inter-area TRPPM. Table 1 shows the numerical characteristics of the inter-area TRPPM. Using the mean and standard deviation, the PDF and cumulative distribution function (CDF) of the inter-area 
TRPPM can be calculated. According to the PDF, TRPPM is reserved for corresponding uncertain power generations. Therefore, the accuracy of the inter-area DPEPOPF mathematical model and its optimization algorithm are verified.

Table 1. Numerical characteristics of the tie-line reserve power probability margin (TRPPM).

\begin{tabular}{cccc}
\hline From-Bus & To-Bus & Mean & Standard Deviation \\
\hline 15 & 33 & -1.36 & 21.17 \\
19 & 34 & -0.68 & 10.66 \\
30 & 38 & -3.12 & 48.77 \\
23 & 24 & -2.65 & 41.38 \\
47 & 69 & -0.14 & 2.15 \\
65 & 68 & -2.39 & 37.38 \\
49 & 69 & -0.12 & 1.85 \\
\hline
\end{tabular}

According to Table 1, the tie-line with the minimum mean and standard deviation is that between bus 49 and bus 69; its PDF and CDF are shown in Figure 8. Under the same uncertain power generation, the probability of the reserved power margin of the tie-line corresponding to the minimum mean and standard deviation is smaller. In the process of accommodating power to participate in dispatching, line power congestion is likely. Therefore, it is meaningful to study the TRPPM for such tie-lines to avoid power congestion.

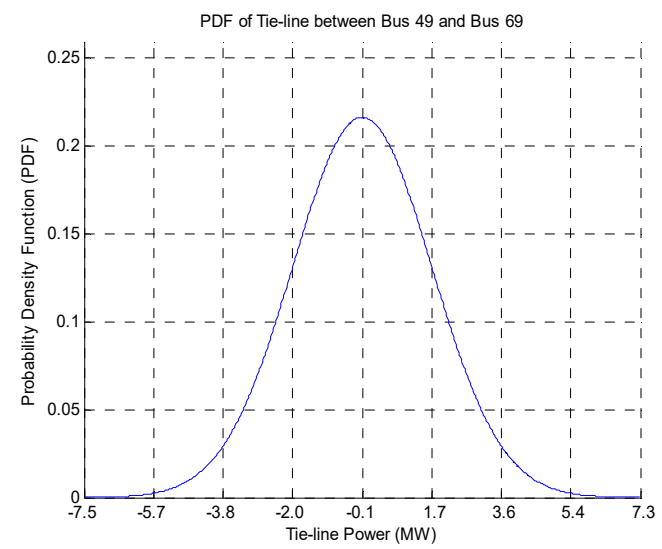

(a)

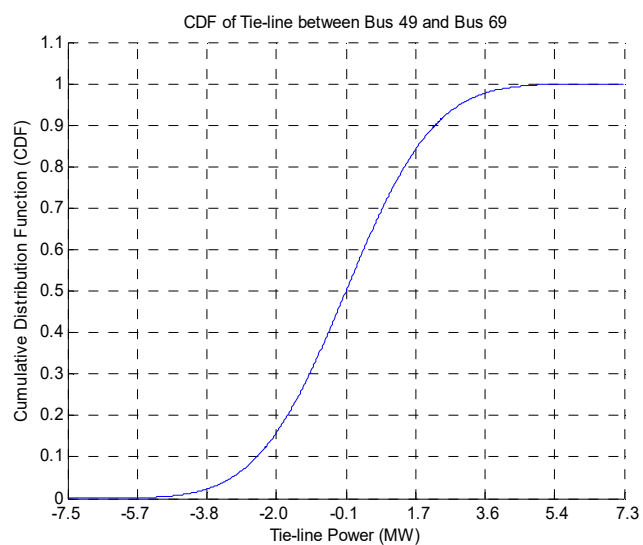

(b)

Figure 8. Probability density function (PDF) and cumulative distribution function (CDF) of the TRPPM between bus 49 and bus 69. (a) PDF; (b) CDF.

\subsection{Simulation Results of Intra-Area TRPPM}

According to Equation (28), the TRPPM in areas 1-3 are calculated based on the intra-area DPEPOPF and its optimization algorithm.

Tables A2-A4 (Appendix B) show the numerical characteristics of the TRPPM calculated by simulation in power grid areas 1-3, respectively. Using the mean and standard deviation, the PDF and CDF of intra-area TRPPM can be calculated. In day-ahead dispatching, according to the PDF of TRPPM, different sizes of the TRPPM are reserved for different magnitudes of uncertain power generation.

For the three regions, Table 2 shows the numerical characteristics of the mean and standard deviation corresponding to the minimum TRPPM. The mean of the TRPPM between bus 18 and bus 19 is the smallest, and the standard deviation of the TRPPM between bus 68 and bus 116 is the smallest. 
Table 2. Numerical characteristics of the mean and standard deviation corresponding to the minimum TRPPM.

\begin{tabular}{ccccc}
\hline Condition & From-Bus & To-Bus & Mean & Standard Deviation \\
\hline Minimum mean & 18 & 19 & -0.1840 & 22.0614 \\
Minimum standard deviation & 68 & 116 & 0 & 0.0013 \\
\hline
\end{tabular}

Figures 9 and 10, respectively, depict the PDF and CDF of the corresponding TRPPM when the mean and standard deviation are minimum. According to the PDF, the TRPPM is reserved for different magnitudes of uncertain power generation. Therefore, the accuracy of the intra-area DPEPOPF mathematical model and its optimization algorithm is verified.

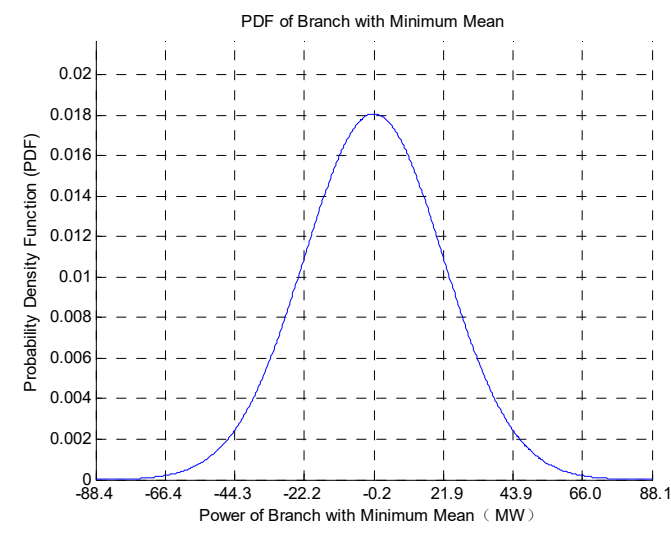

(a)

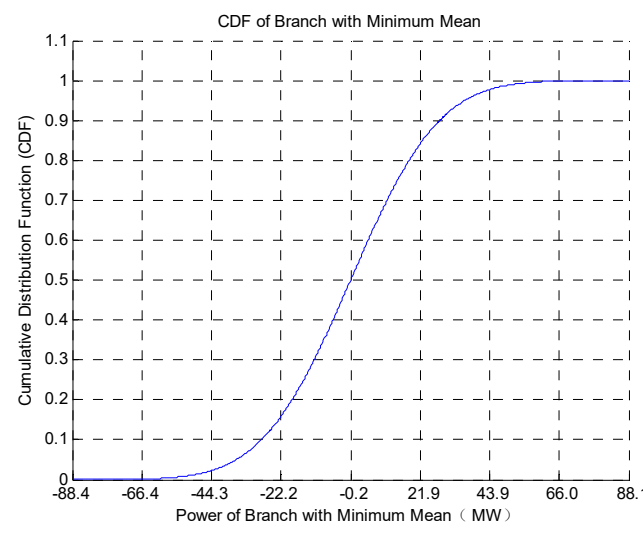

(b)

Figure 9. PDF and CDF of the mean minimum corresponding TRPPM. (a) PDF; (b) CDF.

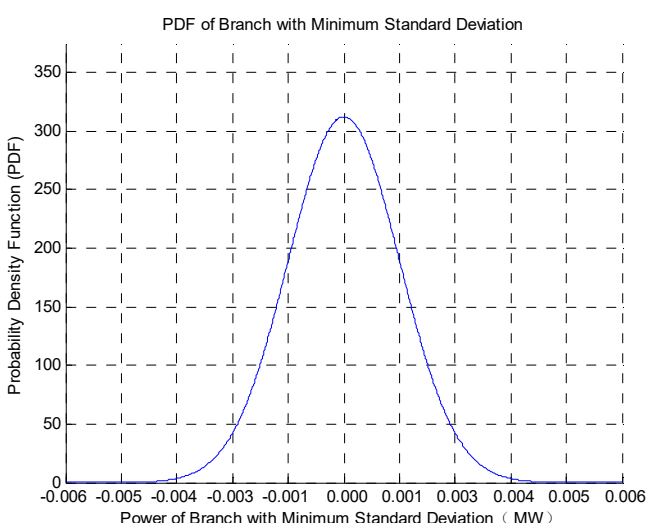

(a)

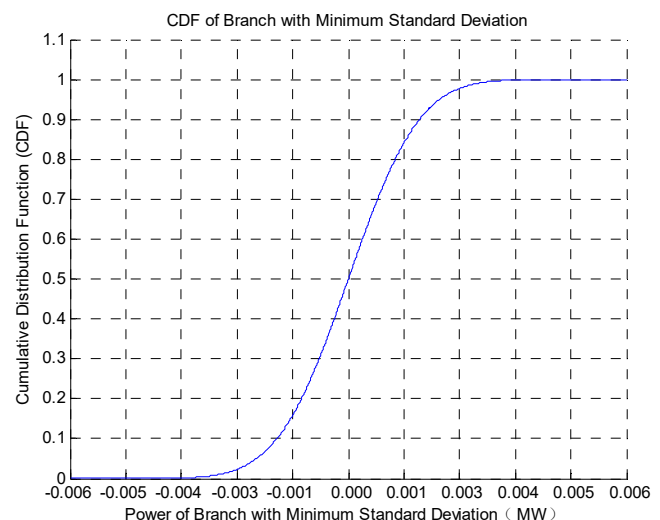

(b)

Figure 10. PDF and CDF of the standard deviation minimum corresponding to the TRPPM. (a) PDF; (b) CDF.

\subsection{Discussion}

Equations (6) and (9) are DC power flow model equations. Equations (7) and (10) are AC power flow model equations. Therefore, the DPEPOPF mathematical model is a combination of AC power flow and DC power flow. In reference [48], a probabilistic optimal DC power flow model is proposed and solved by the method of combined cumulants and Gram-Charlier expansion. In this paper, the calculation results of the DPEPOPF model and the DC power flow model were compared with the AC power flow model, respectively, to verify that the calculation results of the power flow model proposed in this paper are more accurate than the DC power flow model, and approximate to the AC model 
results. In this paper, the operation time of the DPEPOPF model, the DC power flow model, and the AC power flow model were compared to verify that the operation speed of the model proposed in this paper is faster than that of the AC model, and is similar to that of the DC model.

In order to verify the accuracy of the calculation results of the power flow model proposed in this paper, the calculation results of the DPEPOPF model and the DC power flow model were compared with the AC power flow model, respectively. The relative error of the calculation results of the two models relative to the AC power flow model is defined by Equation (39):

$$
\text { error }=\frac{\left|P_{A C}-P_{L}\right|}{P_{A C}} \times 100 \%
$$

where error represents the percentage error of the simulation result; $P_{A C}$ represents the simulation result of the AC power flow model; $P_{L}$ represents the simulation result of the DPEPOPF mathematical model or DC power flow model. The average percentage error $\overline{\text { error }}$ is calculated by Equation (40):

$$
\overline{\text { error }}=\frac{\sum \text { error }}{N_{L}}
$$

where $N_{L}$ represents the total number of percentages error.

According to Table 3, the average relative error of the DPEPOPF and the AC power flow calculation result is $0.396 \%$; the average relative error of the DC power flow and the AC power flow calculation result is $11.05 \%$. The simulation results show that the results of the optimal power flow model proposed in this paper are similar to those of the AC power flow model, and more accurate than those of the DC power flow model. What is more, the proportion of uncertain power source in PSHPRPSs is higher, and the day-ahead prediction error of renewable energy sources is generally about $25 \%$. According to Table 3, the average relative error of the DPEPOPF and the AC power flow calculation result is $0.396 \%$, which is much smaller than the power prediction error in PSHPRPSs. Therefore, the simulation results prove the accuracy of the DPEPOPF model proposed in this paper.

Table 3. Average percentage error of simulation results. DC, direct current.

\begin{tabular}{ccc}
\hline Power Flow Model & DPEPOPF Model & DC Power Flow Model \\
\hline$\overline{\text { error }}$ & $0.396 \%$ & $11.05 \%$ \\
\hline
\end{tabular}

In order to verify the efficiency of the proposed model, the operation time of the optimal power flow model, AC power flow model, and DC power flow model are compared.

As shown in Table 4, the operation time of the DPEPOPF model is shorter than that of the AC power flow, and similar to that of the DC power flow. Therefore, the simulation results show that the proposed model has high computational efficiency.

Table 4. Simulation operation time. AC, alternating current.

\begin{tabular}{cccc}
\hline Model & DPEPOPF Model & DC Power Flow Model & AC Power Flow Model \\
\hline Operation time (s) & 0.076 & 0.023 & 0.270 \\
\hline
\end{tabular}

The simulation results show that the proposed probabilistic optimal power flow model not only has high calculation accuracy, but also has high calculation efficiency.

Large-scale renewable energy sources and main loads are usually distributed in different power grid areas. The power generated by large renewable energy sources needs to be transmitted via a tie-line to other grid areas where the load is concentrated. Therefore, to accommodate the uncertain power generation of large renewable energy sources, it is necessary to reserve a reserve power probability margin for inter-area tie-lines. The simulation results presented in Section 4.2 show the solution of 
TRPPM of inter-area tie-lines, which was obtained using the inter-area DPEPOPF and its optimization algorithm. To accommodate the intra-area uncertain power generation, it is necessary to reserve a reserve power probability margin for the intra-area tie-lines. The simulation results in Section 4.3 show the solution of TRPPM of intra-area tie-lines, which was obtained using the intra-area DPEPOPF and its optimization algorithm. As the uncertain power generation cannot be predicted in advance, in order to avoid the impact of tie-line congestion on the real-time dispatching and to accommodate uncertain power generation, this study calculated the PDF and CDF of the TRPPM for each tie-line. Thus, the TRPPM is reserved for uncertain power generation with different probabilities. Based on the simulation results presented in Sections 4.2 and 4.3, the model proposed in this paper and its optimization algorithm can effectively reserve the TRPPM for each tie-line. Based on the comparison of DPEPOPF mathematical model and AC power flow simulation results, the accuracy of the DPEPOPF mathematical model and its optimization algorithm is verified.

\section{Conclusions}

To avoid tie-line power congestion in the process of accommodating uncertain power generation in PSHPRPSs, this study developed a DPEPOPF. To avoid uncertain power generation affecting the external power grid, this study adopted the principle of area control error accommodation and divides the power grid area according to the properties of the power source. Based on the grid area division, the DPEPOPF was divided into two categories: An inter-area DPEPOPF mathematical model and an intra-area DPEPOPF mathematical model. The mathematical model was solved using the point estimation method of probabilistic optimal power flow, and the mean and standard deviation of TRPPM for each tie-line are obtained. Furthermore, the PDF and CDF of the TRPPM were obtained. To avoid tie-line power congestion, this model can reserve different TRPPM for different uncertain power generation. The simulation results verify the accuracy of the model and effectively avoid power congestion in the tie-line. For day-ahead dispatching with low accuracy requirements, this model significantly improves operation efficiency.

Author Contributions: Conceptualization, Y.C. and Z.G.; methodology, Y.C.; software, Y.C.; validation, Y.C. and Z.G.; formal analysis, A.T.T.; investigation, H.L.; resources, G.W.; data curation, Y.H.; writing-original draft preparation, Y.C.; writing-review and editing, A.T.T.; visualization, Y.H.; supervision, H.L.; project administration, G.W.

Funding: This research was funded in part by the National Natural Science Foundation of China (grant number 51877047).

Conflicts of Interest: The authors declare no conflicts of interest.

\section{Nomenclature}

PSHPRPSs Power systems with a high proportion of renewable power sources

DPEPOPF Day-ahead prediction error probability optimal power flow

TRPPM Tie-line reserve power probability margin

DCS Dispatch control system

AGC Automatic generation control

CDF Cumulative distribution function

PDF Probability density function

IEEE Institute of Electrical and Electronics Engineers

DC Direct current

REI Radial equivalent independent

AC Alternating current

$M_{l} \quad$ Equivalent bus

$P_{g} \quad$ Active power of the equivalent bus

$P_{g, i} \quad$ Active power of each power source

$P_{l, i} \quad$ Power of each load 
$P_{i j} \quad$ Total power of tie-line

$P_{i j, t} \quad$ Power of $t$ th tie-line

$X_{i j, t} \quad$ Reactance of the $t$ th tie-line

$\Delta P_{Z I} \quad$ Power variation of equivalent bus

$P_{Z S} \quad$ Active power of the equivalent bus

$\Delta \theta_{Z I} \quad$ Phase angle variable of the equivalent bus

$P_{Z I}^{0} \quad$ Calculated value of equivalent bus active power

$y_{i 0} \quad$ Admittance between bus $i$ and the zero-potential bus

$G_{i j} \quad$ Conductance between bus $i$ and bus $j$

$B_{i j} \quad$ Represents the reactance between bus $i$ and bus $j$

$\Delta P_{Z L} \quad$ Power margin of inter-area tie-line

$\sum \Delta P_{f} \quad$ Day-ahead power prediction error

$\sum P_{r} \quad$ Reserve power

$\Delta P_{Z l, i m a x} \quad$ Line residual power margin

$P_{Z R, i} \quad$ Reserve power of the $i$ th power grid area

$g\left(P_{Z R, i}\right) \quad$ Reserve power function

$\sum P_{R, \mathrm{I}}$

$\sum P_{R, I I I}$

$\Delta P_{Z f i}$

$\eta_{Z i, j}$

Accommodation capacity of a type I grid

Accommodation capacity of a type III grid

$M\left(\Delta P_{Z f i}\right)$

Uncertain power generation of the $i$ th regional power grid

$\mu$

Standard location coefficient

$\mu$ Mean

$\sigma_{Z i} \quad$ Standard deviation

$f\left(\Delta P_{Z f i}\right) \quad$ PDF of the power grid area uncertainty power generation

$\Delta P_{Z f} \quad$ Power prediction error of uncertainty power source

$\triangle P_{Z l \max } \quad$ Inter-area tie-line residual power margin

$\sum \Delta P_{I} \quad$ Intra-area uncertain generating power

$\sum P_{R, I} \quad$ Total reserve power

error Percentage error of the simulation result

$P_{A C} \quad$ Simulation result of the AC power flow model

$P_{L} \quad$ Simulation result of the DPEPOPF mathematical model

$\overline{\text { error }} \quad$ Average percentage error

\section{Appendix A}

Appendix A presents the numerical characteristics of uncertain power source prediction errors for the modified IEEE 118 bus test system (see Section 4.1 for details).

Table A1. Numerical characteristics of uncertain power source prediction errors for the modified IEEE 118 bus test system.

\begin{tabular}{cccc}
\hline Bus & Mean & Standard Deviation & Area \\
\hline 12 & 0.0555 & 0.407 & 1 \\
25 & 0.096 & 0.64 & 1 \\
26 & 0.0828 & 0.7452 & 1 \\
36 & 0.015 & 0.18 & 2 \\
42 & 0.03 & 0.205 & 2 \\
55 & 0.02 & 0.20 & 2 \\
59 & 0.102 & 0.51 & 2 \\
66 & 0.0984 & 0.984 & 2 \\
74 & 0.02 & 0.20 & 3 \\
80 & 0.1154 & 1.154 & 3 \\
91 & 0.015 & 0.18 & 3 \\
100 & 0.1056 & 0.704 & 3 \\
105 & 0.035 & 0.195 & 3 \\
110 & 0.04 & 0.17 & 3 \\
112 & 0.025 & 0.205 & 3 \\
\hline
\end{tabular}




\section{Appendix B}

Appendix B presents the simulation results of the modified IEEE 118 bus test system intra-area TRPPM (see Section 4.3 for details).

Table A2. Numerical characteristics of the TRPPM in area 1.

\begin{tabular}{cccccccc}
\hline From-Bus & To-Bus & Mean & Standard Deviation & From-Bus & To-Bus & Mean & Standard Deviation \\
\hline 2 & 1 & 0.60 & 5.28 & 20 & 19 & 1.58 & 17.31 \\
3 & 1 & -0.59 & 4.72 & 15 & 19 & -1.40 & 10.62 \\
5 & 4 & -2.45 & 5.25 & 21 & 20 & 1.58 & 17.31 \\
5 & 3 & -1.35 & 0.64 & 22 & 21 & 1.58 & 17.31 \\
5 & 6 & -2.46 & 5.37 & 23 & 22 & 1.58 & 17.31 \\
6 & 7 & -2.46 & 5.37 & 25 & 23 & 3.56 & 34.59 \\
9 & 8 & -23.43 & 26.57 & 26 & 25 & -3.39 & 4.66 \\
8 & 5 & -9.02 & 3.65 & 25 & 27 & 2.65 & 24.75 \\
10 & 9 & -23.43 & 26.57 & 27 & 28 & 1.02 & 10.15 \\
4 & 11 & -2.45 & 4.75 & 28 & 29 & 1.02 & 10.15 \\
5 & 11 & -2.76 & 4.18 & 30 & 17 & -2.74 & 38.83 \\
11 & 12 & -4.05 & 15.26 & 30 & 8 & 14.41 & 32.92 \\
12 & 2 & 0.60 & 5.28 & 26 & 30 & 11.67 & 79.18 \\
12 & 3 & 0.76 & 4.08 & 17 & 31 & -2.42 & 14.71 \\
7 & 12 & -2.46 & 5.37 & 31 & 29 & -1.02 & 10.15 \\
11 & 13 & -1.16 & 6.34 & 23 & 32 & 1.98 & 17.28 \\
12 & 14 & -1.08 & 8.70 & 32 & 31 & 1.39 & 14.57 \\
15 & 13 & 1.16 & 6.34 & 27 & 32 & 1.07 & 9.62 \\
15 & 14 & 1.08 & 8.70 & 113 & 17 & 2.21 & 7.31 \\
16 & 12 & 1.23 & 2.01 & 32 & 113 & 2.21 & 17.31 \\
17 & 15 & 0.84 & 40.80 & 32 & 114 & -0.55 & 4.98 \\
17 & 16 & 1.23 & 2.01 & 27 & 115 & 0.55 & 4.98 \\
17 & 18 & -0.18 & 22.06 & 114 & 115 & -0.55 & 4.98 \\
18 & 19 & -0.18 & 22.06 & 12 & 117 & 0.00 & 0.00 \\
\hline
\end{tabular}

Table A3. Numerical characteristics of the TRPPM in area 2.

\begin{tabular}{cccccccc}
\hline From-Bus & To-Bus & Mean & Standard Deviation & From-Bus & To-Bus & Mean & Standard Deviation \\
\hline 36 & 35 & 0.78 & 1.81 & 49 & 54 & -2.48 & 22.40 \\
37 & 35 & -0.78 & 1.81 & 54 & 55 & -0.64 & 6.03 \\
37 & 33 & 0.00 & 0.00 & 54 & 56 & -1.41 & 12.95 \\
36 & 34 & 0.72 & 16.19 & 56 & 55 & -2.10 & 20.03 \\
37 & 34 & -2.90 & 34.62 & 57 & 56 & -1.22 & 11.03 \\
38 & 37 & 10.80 & 36.20 & 50 & 57 & -1.22 & 11.03 \\
37 & 39 & 7.28 & 1.70 & 58 & 56 & -1.02 & 9.18 \\
37 & 40 & 7.21 & 10.01 & 51 & 58 & -1.02 & 9.18 \\
39 & 40 & 7.28 & 10.05 & 59 & 54 & 0.89 & 7.55 \\
40 & 41 & -6.02 & 10.01 & 59 & 56 & 1.55 & 13.14 \\
42 & 40 & 6.04 & 0.81 & 59 & 55 & 0.73 & 6.05 \\
42 & 41 & 6.02 & 0.81 & 60 & 59 & -1.31 & 4.54 \\
44 & 43 & 2.18 & 0.81 & 61 & 59 & -1.35 & 4.67 \\
34 & 43 & -2.18 & 12.73 & 61 & 60 & -0.91 & 3.11 \\
45 & 44 & 2.18 & 20.81 & 62 & 60 & -0.40 & 1.43 \\
46 & 45 & 0.95 & 16.46 & 61 & 62 & 0.26 & 1.02 \\
47 & 46 & 0.53 & 20.81 & 63 & 59 & -4.37 & 15.04 \\
48 & 46 & 0.42 & 16.46 & 64 & 63 & -4.37 & 15.04 \\
49 & 47 & 0.53 & 20.81 & 64 & 61 & -2.00 & 6.76 \\
49 & 42 & 9.05 & 0.43 & 65 & 38 & 10.80 & 36.20 \\
49 & 45 & 1.23 & 11.92 & 65 & 64 & -6.37 & 21.80 \\
49 & 48 & 0.42 & 16.46 & 66 & 49 & 6.06 & 86.46 \\
49 & 50 & -1.22 & 11.03 & 66 & 62 & -0.33 & 1.23 \\
49 & 51 & -1.47 & 13.32 & 67 & 62 & -0.33 & 1.23 \\
51 & 52 & -0.46 & 4.13 & 65 & 66 & -4.43 & 14.40 \\
52 & 53 & -0.46 & 4.13 & 66 & 67 & -0.33 & 1.23 \\
54 & 53 & 0.46 & 4.13 & & & & \\
\hline
\end{tabular}


Table A4. Numerical characteristics of the TRPPM in area 3.

\begin{tabular}{|c|c|c|c|c|c|c|c|}
\hline From-Bus & To-Bus & Mean & Standard Deviation & From-Bus & To-Bus & Mean & Standard Deviation \\
\hline 68 & 69 & 12.60 & 54.03 & 93 & 94 & 0.53 & 17.92 \\
\hline 69 & 70 & 16.15 & 41.86 & 94 & 95 & 4.37 & 11.13 \\
\hline 70 & 24 & 17.80 & 21.16 & 96 & 80 & 2.62 & 5.11 \\
\hline 70 & 71 & 17.80 & 63.24 & 96 & 82 & 4.06 & 33.91 \\
\hline 72 & 24 & 17.80 & 6.75 & 94 & 96 & 4.93 & 12.56 \\
\hline 71 & 72 & 17.80 & 43.25 & 97 & 80 & 2.62 & 5.11 \\
\hline 71 & 73 & 0.00 & 20.00 & 98 & 80 & 4.82 & 12.60 \\
\hline 70 & 74 & -8.99 & 30.68 & 99 & 80 & 4.82 & 12.58 \\
\hline 75 & 70 & 10.45 & 31.86 & 92 & 100 & -1.34 & 21.31 \\
\hline 69 & 75 & 4.73 & 6.75 & 94 & 100 & -8.24 & 59.54 \\
\hline 75 & 74 & 6.99 & 10.68 & 95 & 96 & 4.37 & 11.13 \\
\hline 77 & 76 & 5.64 & 15.89 & 96 & 97 & 2.62 & 5.11 \\
\hline 77 & 69 & 8.28 & 31.25 & 100 & 98 & 4.82 & 12.60 \\
\hline 77 & 75 & 7.07 & 19.91 & 100 & 99 & 4.82 & 12.58 \\
\hline 77 & 78 & -3.27 & 18.04 & 101 & 100 & -1.34 & 21.37 \\
\hline 79 & 78 & 3.27 & 18.04 & 92 & 102 & -1.34 & 21.37 \\
\hline 80 & 77 & 10.56 & 58.29 & 102 & 101 & -1.34 & 21.37 \\
\hline 80 & 79 & 3.27 & 18.04 & 100 & 103 & -5.95 & 33.98 \\
\hline 81 & 68 & 12.60 & 54.03 & 100 & 104 & -2.11 & 11.98 \\
\hline 80 & 81 & 12.60 & 54.03 & 103 & 104 & -0.74 & 4.17 \\
\hline 82 & 77 & 7.18 & 9.29 & 103 & 105 & -1.38 & 7.82 \\
\hline 83 & 82 & 3.11 & 43.20 & 100 & 106 & -1.94 & 11.04 \\
\hline 84 & 83 & 1.34 & 18.58 & 104 & 105 & -2.85 & 16.15 \\
\hline 85 & 83 & 1.78 & 24.62 & 105 & 106 & 1.69 & 9.61 \\
\hline 85 & 84 & 1.34 & 18.58 & 105 & 107 & 0.25 & 1.44 \\
\hline 85 & 86 & 0.00 & 50.00 & 105 & 108 & -2.67 & 15.50 \\
\hline 87 & 86 & 0.00 & 50.00 & 106 & 107 & -0.25 & 1.44 \\
\hline 88 & 85 & 1.56 & 13.39 & 108 & 109 & -2.67 & 15.50 \\
\hline 89 & 85 & 1.56 & 13.41 & 103 & 110 & -3.83 & 22.00 \\
\hline 89 & 88 & 1.56 & 13.39 & 109 & 110 & -2.67 & 15.50 \\
\hline 89 & 90 & -0.99 & 19.04 & 111 & 110 & 0.00 & 0.00 \\
\hline 90 & 91 & -0.99 & 19.04 & 110 & 112 & -2.50 & 20.50 \\
\hline 89 & 92 & -2.13 & 77.48 & 68 & 116 & 0.00 & 0.00 \\
\hline 91 & 92 & 0.51 & 1.04 & 75 & 118 & -5.64 & 15.89 \\
\hline 92 & 93 & 0.53 & 17.92 & 76 & 118 & 5.64 & 15.89 \\
\hline 92 & 94 & 0.53 & 17.92 & & & & \\
\hline
\end{tabular}

\section{References}

1. Wang, Z.; Guo, Z. On Critical Timescale of Real-Time Power Balancing in Power Systems with Intermittent Power Sources. Electr. Power Syst. Res. 2018, 155, 246-253. [CrossRef]

2. Zhang, B.; Wu, W.; Zheng, T.; Sun, H. Design of a Multi-Time Scale Coordinated Active Power Dispatching System for Accommodating Large Scale Wind Power Penetration. Autom. Electr. Power Syst. 2011, 35, 1-6.

3. Zhai, J.; Ren, J.; Zhou, M.; Li, Z. Multi-Time Scale Fuzzy Chance Constrained Dynamic Economic Dispstch Model for Power System with Wind Power. Power Syst. Technol. 2016, 40, 1094-1099.

4. Shang, J. Coordination Model and Algorithm of Energy-Saving Power Generation Dispatching Based on Time Scale. Power Syst. Technol. 2008, 32, 56-61.

5. Happ, H.H. Optimal Power Dispatch-A Comprehensive Survey. IEEE Trans. Appar. Syst. 1977, 96, 841-854. [CrossRef]

6. Wang, K.; Zhang, B.; Yan, D.; Li, Y.; Luo, T. A Multi-Time Scale Rolling Coordination Scheduling Method for Power Grid Integrated with Large Scale Wind Farm. Power Syst. Technol. 2014, 38, 2434-2440.

7. Liu, C.; Chao, Q.; Wei, L. Wind-Storage Coupling Based on Actual Data and Fuzzy Control in Multiple Time Scales for Real-Time Rolling Smoothing of Fluctuation. Electr. Power Autom. Equip. 2015, 35, 35-41.

8. Wang, H.; He, C.; Fang, G.; Fu, L. A Gradual Optimization Model of Dispatching Schedule Taking Account of Wind Power Prediction Error Bands. Autom. Electr. Power Syst. 2011, 35, 131-135.

9. Cheng, C.; Li, G.; Cheng, X.; Shen, J.; Lu, J. Large-Scale Ultra Voltage Direct Current Hydropower Absorption and Its Experiences. Proc. CSEE 2015, 35, 549-560. 
10. Huang, C.; Ding, J.; Tian, G.; Tang, H. Hydropower Operation Modes of Large-Scale Wind Power Grid Integration. Autom. Electr. Power Syst. 2011, 35, 37-40.

11. Zhang, G.; Zhang, B. Optimal Power Flow Approach Consider Secondary Reserve Demand with Wind Power Integration. Autom. Electr. Power Syst. 2009, 33, 25-28.

12. Liu, J.; Yu, J.; Liu, Z. An Intelligent Optimal Dispatch Strategy for Spinning Reserve Coping with Wind Intermittent Disturbance. Proc. CSEE 2013, 33, 163-170.

13. Qadrdan, M.; Wu, J.; Jenkins, N.; Ekanayake, J. Operating Strategies for a GB Integrated Gas and Electricity Network Considering the Uncertainty in Wind Power Forecasts. IEEE Trans. Sustain. Energy 2014, 5, 128-138. [CrossRef]

14. Dai, X.; Deng, Z.; Liu, G.; Tang, X.; Zhang, F.; Deng, Z. Review on Advanced Flywheel Energy Storage System with Large Scale. Trans. China Electrotech. Soc. 2011, 26, 133-140.

15. Hou, Y.; Jiang, X.; Jiang, J. SMES Based Unified Power Quality Conditioner and Control Strategy. Autom. Electr. Power Syst. 2003, 27, 49-53.

16. Huang, X.; Zhang, L.; Zheng, Q.; Alfred, R. Optimization Design of Electricity Saving Device in Hybrid Energy Storage System Based on Compressed Air and Supercapacitors. J. Power Supply 2013, 50, 72-78.

17. Yang, N.; Wang, B.; Liu, D.; Zhao, J.; Wang, H. An Integrated Supply-Demand Stochastic Optimization Method Considering Large-Scale Wind Power and Flexible Load. Proc. CSEE 2013, 33, 63-69.

18. Wang, B.; Liu, X.; Li, Y. Day-Ahead Generation Scheduling and Operation Simulation Considering Demand Response in Large-Capacity Wind Power Integrated Systems. Proc. CSEE 2013, 33, 35-44.

19. Yu, D.; Song, S.; Zhang, B.; Han, X. Synergistic Dispatch of PEVs Charging and Wind Power in Chinese Regional Power Grids. Autom. Electr. Power Syst. 2011, 35, $24-29$.

20. Xiao, X.; Chen, Z.; Liu, N. Integrated Mode and Key Issues of Renewable Energy Sources and Electric Vehicles' Charging and Discharging Facilities in Microgrid. Trans. China Electrotech. Soc. 2013, 28, 1-14.

21. Dong, Z.; Yang, D.; Reindl, T.; Walsh, W.M. Satellite Image Analysis and a Hybrid ESSS/ANN Model to Forecast Solar Irradiance in the Tropics. Energy Convers. Manag. 2014, 79, 66-73. [CrossRef]

22. Ding, M.; Yang, R. Research on Short-Term Prediction of PV Output Power Based on Weather Forecast. Renew. Energy Resour. 2014, 32, 385-391.

23. Dong, Y.; Guo, H.; Wu, S.; Wang, Z.; Ming, F.A.N. Model Prediction Tree for Forecasting Photovoltaic Power Generation. Renew. Energy Resour. 2014, 32, 253-258.

24. Yang, X.; Xiao, Y.; Chen, S. Wind Speed and Generated Power Forecasting Wind Farm. Proc. CSEE 2005, 25, 1-5.

25. Wang, S.; Yu, J. Joint Conditions Probability Forecast Method for Wind Speed and Wind Power. Proc. CSEE 2011, 31, 7-15.

26. Elena Dragomir, O.; Dragomir, F.; Stefan, V.; Minca, E. Adaptive Neuro-Fuzzy Inference Systems as a Strategy for Predicting and Controling the Energy Produced from Renewable Sources. Energies 2015, 8, 13047-13061. [CrossRef]

27. Monteiro, C.; Santos, T.; Fernandez-Jimenez, L.A.; Ramirez-Rosado, I.J.; Terreros-Olarte, M.S. Short-Term Power Forecasting Model for Photovoltaic Plants Based on Historical Similarity. Energies 2013, 6, 2624-2643. [CrossRef]

28. Niya, C.; Zheng, Q.; Ian, T.N. Wind Power Forecasts Using Gaussian Processes and Numerical Weather Prediction. IEEE Trans. Power Syst. 2014, 29, 656-665.

29. Wang, F.; Mi, Z.; Su, S.; Zhao, H. Short-Term Solar Irradiance Forecasting Model Based on Artificial Neural Network Using Statistical Feature Parameters. Energies 2012, 5, 1355-1370. [CrossRef]

30. Das, U.K.; Tey, K.S.; Seyedmahmoudian, M.; Idna Idris, M.Y.; Mekhilef, S.; Horan, B.; Stojcevski, A. SVR-Based Model to Forecast PV Power Generation under Different Weather Conditions. Energies 2017, 10, 876. [CrossRef]

31. Dong, J.; Yang, P.; Nie, S. Day-Ahead Scheduling Model of the Distributed Small Hydro-Wind-Energy Storage Power System Based on Two-Stage Stochastic Robust Optimization. Sustainability 2019, 11, 2829. [CrossRef]

32. Papari, B.; Edrington, C.S.; Bhattacharya, I.; Rudman, G. Effective Energy Management of Hybrid AC-DC Microgrids with Storage Devices. IEEE Trans. Smart Grid 2019, 10, 193-203. [CrossRef]

33. Zhu, J.; Liu, Q.; Xiong, X.; Ouyang, J.; Xuan, P.; Xie, P.; Zou, J. Multi-time-scale robust economic dispatching method for the power system with clean energy. J. Eng. 2019, 2019, 1377-1381. [CrossRef] 
34. Papari, B.; Edrington, C.S.; Kavousi-Fard, F. An Effective Fuzzy Feature Selection and Prediction Method for Modeling Tidal Current: A Case of Persian Gulf. IEEE Trans. Geosci. Remote Sens. 2017, 55, 4956-4961. [CrossRef]

35. Jabr, R.A.; Pal, B.C. Intermittent wind generation in optimal power flow dispatching. IET Gener. Transm. Distrib. 2009, 3, 66-74. [CrossRef]

36. Wang, Z.; Bian, Q.; Xin, H.; Gan, D. A Distributionally Robust Co-Ordinated Reserve Scheduling Model Considering CVaR-Based Wind Power Reserve Requirements. IEEE Trans. Sustain. Energy 2016, 7, 625-636. [CrossRef]

37. Xu, Y.; Hu, Q.; Li, F. Probabilistic Model of Payment Cost Minimization Considering Wind Power and Its Uncertainty. IEEE Trans. Sustain. Energy 2013, 4, 716-724. [CrossRef]

38. Dobson, I.; Greene, S.; Rajaraman, R.; DeMarco, C.L.; Alvarado, F.L.; Glavic, M.; Zhang, J.; Zimmerman, R. Electric Power Transfer Capability: Concepts, Applications, Sensitivity and Uncertainty; PSERC Publication: Tempe, AZ, USA, 2001; pp. 1-34.

39. Zhizhong, G. Power Network Analytic Wheel; Science Press: Beijing, China, 2008; pp. 262-279.

40. Liu, B.; Zhou, J.; Zhou, H.; Liu, K. An Improved Model for Wind Power Forecast Error Distribution. East China Electr. Power 2012, 40, 286-291.

41. Nan, X.; Li, Q. Energy Storage Power and Capacity Allocation Based on Wind Power Forecasting Error Distribution. Electr. Power Autom. Equip. 2013, 33, 117-122.

42. Aidan, T.; Eleanor, D.; Mark, O. Rolling Unit Commitment for Systems with Significant Installed Wind Capacity. In Proceedings of the 2007 IEEE Lausanne Power Tech, Lausanne, Switzerland, 1-5 July 2007.

43. Makarov, Y.V.; Etingov, P.V.; Ma, J.; Huang, Z.; Subbarao, K. Incorporating Uncertainty of Wind Power Generation Forecast into Power System Operation, Dispatch, and Unit Commitment Procedures. IEEE Trans. Sustain. Energy 2011, 2, 433-442. [CrossRef]

44. Pappala, V.S.; Erlich, I.; Rohrig, K.; Dobschinski, J. A Stochastic Model for the Optimal Operation of a Wind-Thermal Power System. IEEE Trans. Power Syst. 2009, 24, 940-950. [CrossRef]

45. Tewari, S.; Geyer, C.J.; Mohan, N. A Statistical Model for Wind Power Forecast Error and its Application to the Estimation of Penalties in Liberalized Markets. IEEE Trans. Power Syst. 2011, 26, 2031-2039. [CrossRef]

46. Morales, J.M.; Perez-Ruiz, J. Point Estimate Schemes to Solve the Probabilistic Power Flow. IEEE Trans. Power Syst. 2007, 22, 1594-1601. [CrossRef]

47. Wang, J.; Shahidehpour, M.; Li, Z. Security-Constrained Unit Commitment with Volatile Wind Power Generation. IEEE Trans. Power Syst. 2008, 23, 1319-1327. [CrossRef]

48. Pei, Z.; Lee, S.T. Probabilistic load flow computation using the method of combined cumulants and Gram-Charlier expansion. IEEE Trans. Power Syst. 2004, 19, 676-682. 